\title{
Measurement of Complex Permittivity using Planar Resonator
} sensor

\author{
Beulah Jackson ${ }^{1}$, T.Jayanthy ${ }^{2}$ \\ '(Research Scholar, Sathyabama University, Chennai, India) \\ ${ }_{2}^{2}$ (Principal, Panimalar Institute of Technology, Chennai, India)
}

\begin{abstract}
A Microstrip loop resonator sensor designed to determine the dielectric constant of a liquid is proposed. The resonator sensor designed for $1 G H Z$ for different structure of loop is described. The sensor is implemented using planar microstrip technology. A dielectric material is placed above microstrip ring resonator. As a consequence the near field of the resonator interacts with the material under test (MUT) and the electrical characteristics of the resonator change in relation to the permittivity of the material. The proposed models are extremely compact and experimentally verified by the measurements for liquid sample. A vector network analyzer is used to measure the shift in resonant frequency and the corresponding amplitude of the transmission parameter for the fundamental mode. The design procedure is described and experimental results are reported validating the sensor performance.
\end{abstract}

Keywords: Frequency shift, Moisture Content, Permittivity, Resonator sensor

\section{INTRODUCTION}

Microwave sensing techniques have found many applications in industrial measurements such as determination of materials permittivity, moisture content or the constituent proportion in materials [1]-[5]. Traditionally, waveguide and coaxial cavity resonators have been used for measuring both low and high loss/dielectric constant materials. However, due to small size, ease of fabrication and low cost, microstrip resonator techniques have become popular.

Microwave dielectric measurement methods are of two types: Non resonant and resonant methods [5][7]. Resonant methods including resonator perturbation methods, have relatively high accuracy than nonresonant ones [8], [9]. The Microwave sensors based on a microstrip ring resonator sensor for the complex permittivity materials have been developed. Different types of loop sensors are analyzed. The designed sensors are based on one wavelength of transmission line. In this paper the design procedure for three structures are given and their performance in determining the permittivity of a dielectric sample in terms of shift in resonant frequency are compared. Permittivity is measured by means of curve fitting equations generated for the resonators.

\section{Comparision Of Microstrip RESONATORS BETWEen Ring, FOLDED AND FraCtal STRUCTURE}

The resonator with length of one wave length at fundamental frequency of $1 \mathrm{GHz}$ in fig. 1 has been studied. A substrate of FR4 ( $\varepsilon r=4.6)$ has been used for all resonators, resulting into following parameters: feed length $=40 \mathrm{~mm}$, Coupling gap $=0.35 \mathrm{~mm}$, Substrate thickness $=1.6 \mathrm{~mm}$, Microstrip line width $=1 \mathrm{~mm}$ and total resonator length $=170 \mathrm{~mm}$. Resonator length is found out by the following.

Where

$$
\lambda_{g}=\frac{\lambda_{0}}{\sqrt{\varepsilon_{e f f}}}
$$

$$
\varepsilon_{e f f}=\frac{\varepsilon_{r}+1}{2}+\frac{\varepsilon_{r}-1}{2}\left[\left(1+12\left(\frac{h}{w}\right)\right)^{\frac{1}{2}}+0.04\left(\left(1-\frac{w}{h}\right)\right)^{2}\right]
$$




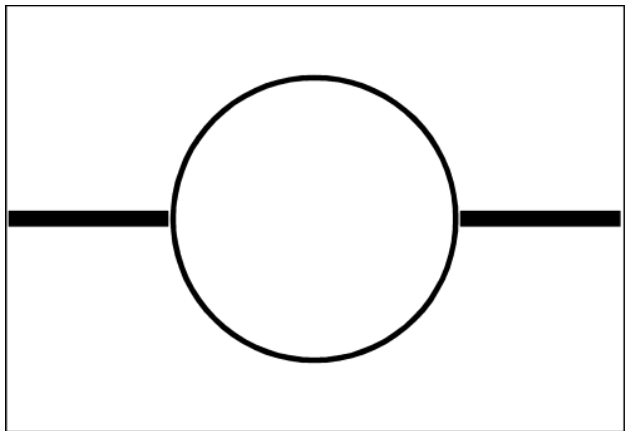

Fig.1 Circular Ring Resonator

Fig.1 illustrates a schematic of the microstrip ring resonator. The resonator responses have been determined by using ADS software. The fundamental resonant frequency is designed at $1 \mathrm{GHz}$. The proposed sensor has been fabricated on a FR4 substrate $\left(\varepsilon_{r}=4.6\right)$ with the dielectric thickness of $1.6 \mathrm{~mm}$. The radius of the ring resonator is $26.5 \mathrm{~mm}$. The strip width of ring resonators is $1 \mathrm{~mm}$ and the coupling gaps are $0.35 \mathrm{~mm}$. For each mode the peak points of insertion loss $\mathrm{S}_{21}$ are recorded.

The folded resonator shown in fig. 2 has been designed at $1 \mathrm{GHz}$ frequency and has a compact size when compared with ring resonator [12] .The resonator layout with all dimensions is shown in Fig. 2 . Wfeed $=2.95 \mathrm{~mm}$, Lfeed $=40 \mathrm{~mm}, \mathrm{~L} 1=5.64 \mathrm{~mm}, \mathrm{~L} 2=23.52 \mathrm{~mm}, \mathrm{r}=5 \mathrm{~mm}$, coupling gap $\mathrm{g}=0.35 \mathrm{~mm}$ and $\mathrm{Zo}=50$ ohms. The physical area of the meander shaped resonator is reduced by $51.58 \%$ of the area of the ring resonator.

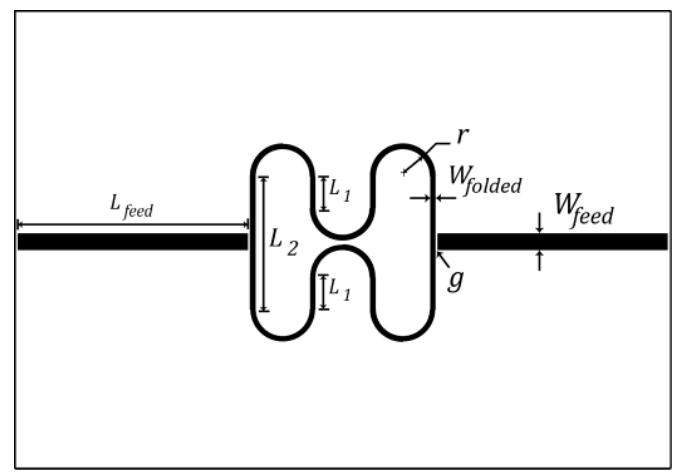

Fig.2 Meander resonator

A Minkowski fractal resonator (fig.3) was designed at resonant frequency of $1 \mathrm{GHz}$ to have a more compact size compared to meander shaped resonator. Applying fractals to resonator elements allows for smaller resonators. A miniaturization of loop resonator using the fractal technique is known as Minkowski square loop resonator [10]. The fractal resonator was created by using the initial square pulse (SP) to iterate at each side of the loop. The starting geometry of the fractal, called the initiator, is a square: Each of the four straight segments of the starting structure is replaced with the generator. The iterative generating procedure continues for an infinite number of times.[11]. The proposed Minkowski square loop resonator with iteration1 is shown in fig.3.In the proposed Minkowski resonator the area has been very much reduced by $24.02 \%$ when compared to ring resonator.

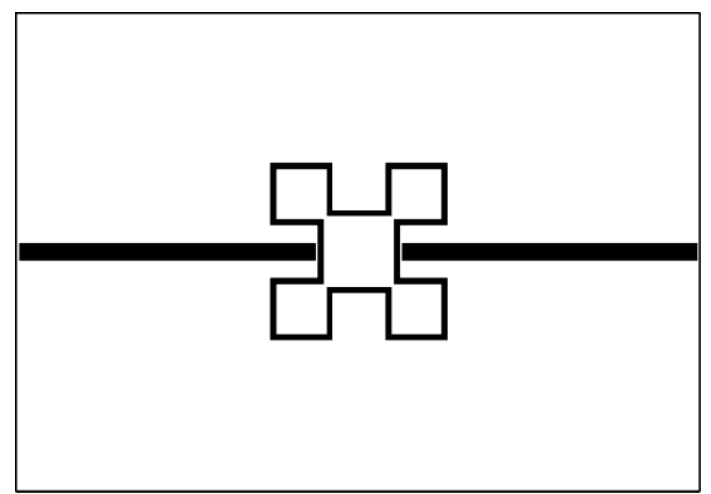

Fig.3Minkowski resonator 
In the above explained resonator sensors a shift in the resonant frequency and insertion loss were observed when palm oil as dielectric was placed over the resonators.

\section{Measurement Setup}

The sample liquid palm oil is placed over the sensor. The shift in the resonant frequency is recorded by feeding probes of the sensor. The signal from the network analyzer is coupled via coaxial cable which connects two ports of the VNA to the sensor probes. The frequency response of the reflected signal is displayed in the network analyzer.

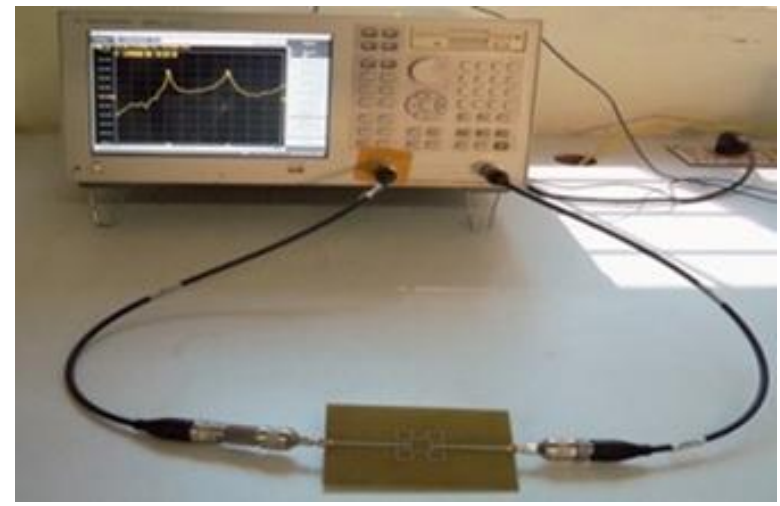

Fig.4 Measurement Setup

The permittivity was determined for mode 1 using the ring, Meander and Minkowski resonators by means of the following polynomial equations generated in curve fitting method.

$$
\begin{array}{ll}
\text { Ring, } & f_{r 1}=1080.96-92.8125 \varepsilon_{r}+9.1941 \varepsilon_{r}{ }^{2}-0.3873 \varepsilon_{r}{ }^{3} \\
\text { Meander, } & f_{r 1}=1103.8-109.5 \varepsilon_{r}+18 \varepsilon_{r}{ }^{2}-1.5 \varepsilon_{r}{ }^{3} \\
\text { Minkowski, } & f_{r 1}=1073.5-68.7314 \varepsilon_{r}+5.2022 \varepsilon_{r}{ }^{2}-0.1876 \varepsilon_{r}{ }^{3}
\end{array}
$$

Where $f_{r 1}$ is shift in frequency

\section{RESUlts}

The measured outputs using network analyzer and the simulated outputs obtained using ADS software are tabulated as in Table.1 and are shown in figures. It is found to be the Minkowski resonator has shown more

\begin{tabular}{|c|c|c|c|c|c|c|c|}
\hline \multirow[b]{2}{*}{ Resonator Type } & \multirow[t]{2}{*}{ Dielectric } & \multicolumn{3}{|c|}{$\begin{array}{c}\text { Simulated Output } \\
\text { Resonant frequency }(\mathrm{GHz})\end{array}$} & \multicolumn{3}{|c|}{$\begin{array}{c}\text { Measured Output } \\
\text { Resonant frequency }(\mathrm{GHz})\end{array}$} \\
\hline & & $\mathrm{n}=1$ & $\mathrm{n}=2$ & $n=3$ & $\mathrm{n}=1$ & $\mathrm{n}=2$ & $n=3$ \\
\hline \multirow{2}{*}{ Ring } & Air & 1 & 2 & 3 & 1.05 & 2.1 & 3.01 \\
\hline & Palm Oil & 0.874 & 1.75 & 2.65 & 0.950 & 1.9 & 2.84 \\
\hline \multirow{2}{*}{ Meander } & Air & 1 & 2 & 3 & 1.05 & 2.1 & 3.01 \\
\hline & Palm Oil & 0.887 & 1.81 & 2.63 & 0.938 & 1.938 & 2.838 \\
\hline \multirow{2}{*}{ Minkowski } & Air & 1 & 2 & 3 & 1.05 & 2.1 & 3.01 \\
\hline & Palm Oil & 0.910 & 1.766 & 2.62 & 0.972 & 1.908 & 2.84 \\
\hline
\end{tabular}
sensitivity compared to the other two resonators.

Table 1: Results

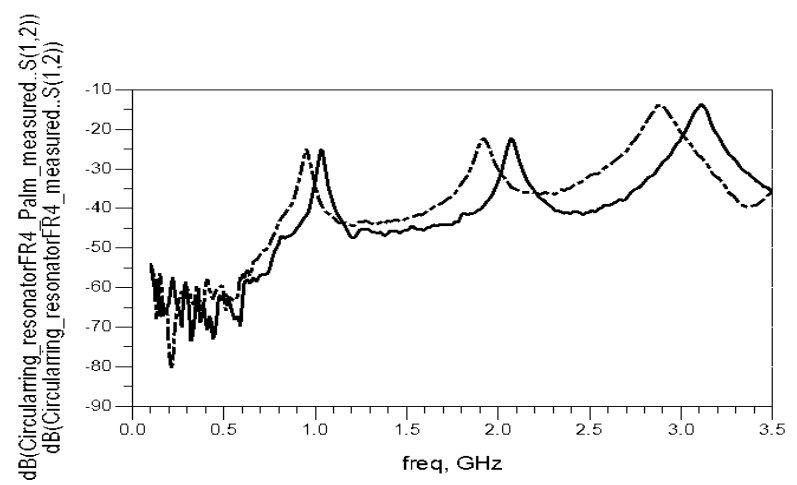

Fig.5 (a) Measured output for ring resonator 


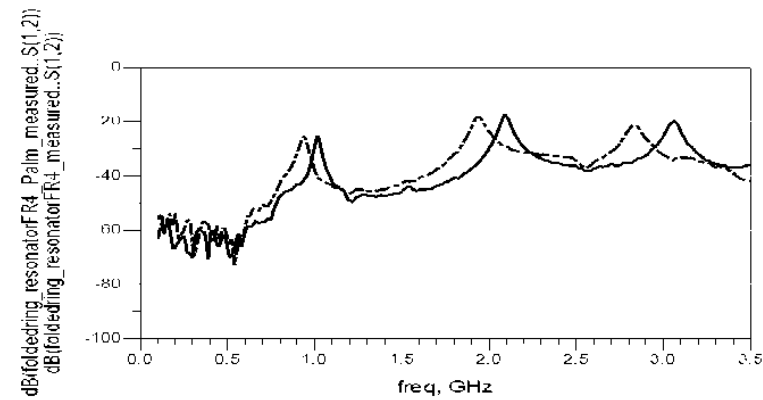

Fig.5 (b) Measured output for Meander resonator

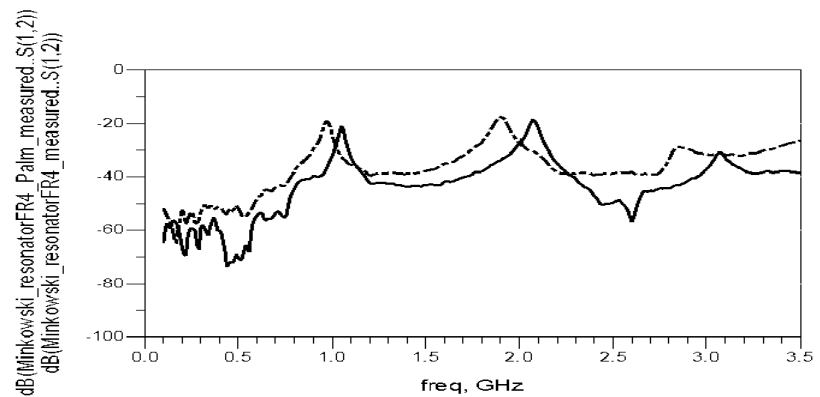

Fig.5(c) Measured output for Minkowski resonator

Since the dielectric constant of any material affects the resonant frequency of the conductor, the permittivity of the test material is determined with the relative shift in frequency. The inversion of the polynomial equations (3)-(5) gives the dielectric constant of the MUT which is given in Table.2.Minkowski resonator gives the nearest value of dielectric constant of palm oil $\left(\varepsilon_{r}=2.8\right)$

Table 2. Dielectric Constant Vs Frequency

\begin{tabular}{|c|c|c|}
\hline Resonator Type & Frequency Shift (MHz) & Dielectric Constant \\
\hline Ring & 900 & 2.5 \\
\hline Meander & 917 & 2.54 \\
\hline Minkowski & 922 & 2.7 \\
\hline
\end{tabular}

\section{CONCLUSION}

For determining dielectric constant of palm oil, the circular, Meander and Minkowski fractal resonator sensor structures have been analyzed and their performance in terms of shift in resonant frequency and insertion loss have been compared. Of all three sensors the fractal resonator is found to have a reduced area when compared to the conventional ring resonator and has less insertion loss. The sensors discussed were simulated, fabricated and tested using Vector network analyzer. The Minkowski fractal resonator sensor has high performances with low cost, compact size and more accuracy, hence can be used for dielectric constant measurement of liquids and solid materials.

\section{REFERENCES}

[1] Nyfors and P. Vainikainen, Industrial Microwave Sensors.Norwood, MA: Artech House, 1989

[2] R. J. King, "Microwave sensors for process control-Part I: Transmission sensors," Sensors, pp. 68-74, September 1992.

[3] M. Kent, "The use of strip-line configuration in microwave moisture Measurement," J. Microw. Power, vol. 7, pp. 185-193, 1972.

[4] W. S. Holmes, S. G. Riley, and A. D. Green, "In-kiln moisture content measurement of timber using a waveguide aperture array," in Proc. IMPISymp., Boston, MA, 1996

[5] W. S. Holmes and S. G. Riley, "Microwave method for In-kiln moisture content measurement," in Proc. IUFRO Wood Drying Conf., Quebec,PQ, Canada, August 1996.

[6] 'Sensors' IEE proc.135,pt.H, No.3(1988)187-195

[7] K.Joshi,R.D.Pollard,"Microstrip resonator technique for non-destructive complex permittivity / moisture measurement”.proc .IEEE MTT/International microwave symposium. Vol.14,issue June 1999.pg1863-1866.

[8] K.Fidanboylu, S.M. Riad, and A. Elshabini-Riad, "A new time-domain approach for determining the complex permittivity using stripline geometry,” IEEE Trans. Instrum. Meas., vol. 39, no. 6, pp.940-944,Dec. 1990

[9] A.K.Verma,Nasimuddin ,A.S.Omar "Microstrip resonator sensors for determination of complex permittivity of material in sheet, liquid and paste forms",IEEE Proc.-Microw. Antennas Propag., vol.152, no.1, Feb. 2005

[10] Gianvittorio, J. P. andY. Rahmat-Samii, "Fractal antennas: A novel antenna miniaturization technique, and applications," IEEE Antennas Propagation Magazine, Vol. 44, No. 1, 20-36, 2002.

[11] Saritha Bajaj, Ajay Koushik"Design and Performance Analysis of Minkowski Square Loop Fractal Antenna" in IJERA ISSN: 2248-9622 Vol. 2, Issue 1, Jan-Feb 2012, pp.229-233

[12] Somporn Seewattanapon "A Microstrip Folded Resonator Sensor for Measurement of Dielectric Constant" Proceedings of ECTI-CON 2008 . 